\title{
Elinor Ostrom et la Gouvernance Economique
}

\author{
Guillaume HOLLARD* \\ Omar SENE*
}

Janvier 2010

Le 12 octobre 2009, le Prix Nobel d'Economie fût décerné aux professeurs Elinor Ostrom et Oliver Williamson pour leurs travaux sur la "gouvernance économique". Elinor Ostrom devenait dès lors la première femme à être récompensée par le Nobel d'économie. Selon les termes employés par le comité, elle a été primée pour "avoir démontré comment les copropriétés peuvent être efficacement gérées par des associations d'usagers " remettant ainsi en cause " l'idée classique selon laquelle la propriété commune est mal gérée et doit être prise en main par les autorités publiques ou le marché". Les travaux d'Ostrom sont encore mal connus de la communauté des économistes universitaires. Deux raisons peuvent être avancées pour expliquer cette relative ignorance. Tout d'abord, l'œuvre d'Elinor Ostrom est souvent cataloguée comme relevant des sciences politiques. Mais, plus profondément, les méthodes utilisées par Ostrom tout au long de sa carrière ne correspondent pas toujours aux canons en vigueur parmi les économistes. Plus précisément, ce qui fait la spécificité de son œuvre, c'est la manière dont elle parvient à faire dialoguer des résultats issus de méthodes d'investigation qui ne sont que rarement combinées dans la pratique, telles que l'étude de cas, l'économie expérimentale ou bien la théorie des jeux. Aussi, cet article se propose de dresser un panorama des travaux réalisés par Ostrom durant ces cinquante dernières années. Nous commencerons par retracer son itinéraire académique et professionnel. Dans une deuxième partie, nous montrerons comment Ostrom mobilise successivement différentes méthodes, toujours au service de l'analyse des biens collectifs.

Nous verrons comment elle parvient à mobiliser successivement différentes méthodes d'investigation tout au long d'un itinéraire qui part de l'étude minutieuse de multiples situations concrètes pour évoluer vers des recommandations de politiques publiques pour le compte de grands organismes internationaux. En conclusion, nous

\footnotetext{
*Ecole d'économie de Paris et Centre d'économie de la Sorbonne, Université de Paris 1.
} 
évoquerons les implications qu'elle tire de son travail pour contribuer à un renouvellement des théories de la rationalité.

\section{1- Itinéraire académique}

Elinor Ostrom est née en 1933, à Los Angeles, au milieu de la Grande Dépression. Après ses études secondaires au collège Beverly Hills High School, elle intègre I'Université de Californie à Los Angeles (UCLA) pour suivre des études en sciences politiques. Elle obtient une licence en sciences politiques, en 1954, avant de migrer à Boston où elle travaille dans un cabinet d'avocat. Trois ans après, Ostrom revient à Los Angeles pour poursuivre ses études à l'université où elle obtient son master en 1962. Elle finit ses études à l'université de Californie, en 1965, par la présentation de sa thèse de doctorat intitulée "Public Entrepreneurship: A Case Study in Ground Water Management" (Ostrom (1965)). L'obtention de son doctorat lui ouvre les portes de l'Université d'Indiana où elle rejoint son mari Vincent Ostrom et débute sa carrière universitaire. Elle est successivement nommée professeur de sciences politiques en 1974, puis directrice du département entre 1980 et 1984 avant d'occuper la chaire de sciences politiques Arthur F. Bentley.

\subsection{Parcours de recherche}

Durant toute sa carrière, Ostrom s'est consacrée presque exclusivement à l'étude de la gestion collective des ressources. Elle a commencé, dans le cadre de sa thèse de doctorat, par étudier comment les associations d'usagers arrivaient, par des arrangements institutionnels, à gérer l'intrusion de l'eau salée dans la nappe phréatique à Los Angeles. Après cette première expérience, elle se voit confier une étude sur l'organisation de la police à Indianapolis et ses conclusions l'amèneront à contester l'efficacité de la centralisation des commissariats, analyse qu'elle élargira dans le domaine de l'action collective. En 1973, soucieuse de dépasser les frontières disciplinaires, elle fonde, avec son mari, l'atelier "Political Theory et Policy Analysis" visant à fournir un cadre intellectuel pour ses recherches. II s'agira d'identifier les institutions les plus adaptées à une gestion efficace de ressources collectives telles que l'eau ou les forêts. Plutôt que de rechercher des règles qui s'appliqueraient indépendamment du contexte, une grande partie de son effort intellectuel sera dédié 
à identifier les éléments du contexte qui doivent être pris en compte pour l'élaboration de principes de fonctionnement.

Ostrom dirigera ensuite de nombreux projets de recherches pour diverses administrations américaines et organismes internationaux dont les études réalisées sont alimentées par ses expériences et les informations relevées dans de nombreux pays d'Amérique, d'Asie, d'Afrique ou d'Europe. Ces nombreuses études de cas lui permettront, plus tard, de développer un vaste programme de recherche à la fois sociologique, anthropologique et économique qui aboutira à la publication, en 1990, de son ouvrage "Gouverning the commons". Les résultats de ses études permettent de mettre en avant l'existence d'une troisième voie, entre le marché et l'Etat, pour la gestion des ressources communes. Elle montre à travers de multiples exemples que les communautés locales peuvent parvenir par elle-même à mettre en place une gestion efficiente, sans avoir recours aux autorités publiques ou au marché. Un point de vue qu'elle n'hésite pas endosser très ouvertement : "if this study does nothing more than shatter the convictions of many policy analysts that the only way to solve common pool resource problems is for external authorities to impose full private property rights or centralized regulation, it will have accomplished one major purpose "†. En 1992, l'Organisation des Nations Unis pour l'Alimentation et l'Agriculture (FAO) lui confie une étude sur les ressources forestières et, depuis lors, elle consacre beaucoup de son temps au programme International Forestry Resources and Institutions (IFRI). Spécialiste des institutions en économie, Ostrom fonde et dirige le Centre pour l'Etude de la Diversité Institutionnelle à l'université d'Etat d'Arizona.

En delà de ses activités de recherches, Ostrom a occupé des postes importants dans de prestigieuses associations. Elle a, entre autres, présidé la Association Américaine de Science Politique. L'accumulation des honneurs académiques ne doit cependant pas masquer le fait qu'Ostrom fait partie des rares intellectuels qui auront connu de leur vivant une large mise en œuvre de leurs idées.

\subsection{Les travaux primés}

L'attribution du prix Nobel d'économie à Ostrom marque la consécration de ses travaux sur la gouvernance. S'appuyant sur de nombreuses études de cas, elle propose une approche originale de la gouvernance des ressources communes et

\footnotetext{
${ }^{\dagger}$ Ostrom (1990), p. 182
} 
démontre que les communautés sont capables de s'autogouverner et d'éviter la surexploitation des ressources. Le problème de la surexploitation des ressources communes, désormais bien connu en économie, avait été analysé, en premier lieu, par Warming (1911) et Gordon (1957) mais fût popularisé par un biologiste, Hardin (1968), sous le nom de " la tragédie des biens communs " dans un article paru dans Science. Le problème classique de " la tragédie des communs " est que chaque usager d'une ressource commune, s'il ne fie qu'à son intérêt individuel, va essayer d'utiliser la ressource de façon à maximiser ses gains individuels, augmentant ainsi le coût pour l'ensemble de la collectivité. C'est cette tension entre intérêt individuel et intérêt collectif qui caractérise les situations dites de dilemmes sociaux (à l'instar du dilemme du prisonnier). Hardin cite l'exemple d'un pâturage communal où chaque éleveur a intérêt à augmenter le nombre de bêtes dans la mesure où le champ ne lui appartient pas et que le coût d'usage est partagé par tout le monde. La combinaison des intérêts individuels aboutit alors à une surexploitation et une dégradation de la ressource. Pour apporter une solution à cette tragédie, deux solutions ont été proposées par les économistes.

La première solution suggère une privatisation des ressources communes. Cette solution repose sur l'idée selon laquelle en convertissant les ressources communes en propriétés privées, les propriétaires seront incités à avoir une gestion rationnelle, au sens où ils internaliseront les externalités. Une solution alternative, souvent associée à Pigou (1920), est de confier la gestion des ressources à l'Etat. Celui-ci prélèvera des taxes, appelées taxes pigouviennes, et définira les droits d'accès. Coase (1960) conteste cette solution qu'il considère comme purement théorique. En effet, selon Coase, l'instauration d'une taxe pigouvienne n'est efficace qu'en absence de coûts de transactions or, en pratique, toute forme de gouvernance possède des coûts de transactions qui peuvent être particulièrement élevés

Dans "Gouverning the commons", Ostrom (1990) propose une troisième voie consistant à laisser les usagers créer leurs propres systèmes de gouvernance. Ainsi, Ostrom, à travers de multiples études de cas, montre que de nombreuses communautés à travers le monde parviennent en pratique à éviter la tragédie des communs, alors que la propriété de ces biens n'est ni privée, ni publique, mais collective. En particulier, ces communautés parviennent à gérer durablement les ressources en créant des institutions à petite échelle bien adaptées aux conditions locales. A l'inverse, elle recense également des cas où les dispositifs mis en place ne 
parviennent pas à freiner la surexploitation des ressources communes. II en résulte un programme de recherches sur la nature des dispositifs institutionnels les plus à même de favoriser une gestion efficace, programme auquel elle restera attachée toute sa vie.

\section{Méthodes et résultats}

Une des spécificités de l'œuvre d'Ostrom est de s'être affranchie avec succès des frontières disciplinaires. Elle mobilise une large gamme de disciplines (théorie économique, science politique, psychologie évolutionniste, anthropologie culturelle, etc.) et emploie diverses méthodes (expériences de laboratoire, études de terrain, théorie des jeux évolutifs, etc.) pour analyser l'action collective. Dans sa phase initiale, son travail est essentiellement inductif et elle adopte volontiers un raisonnement "bottom-up" c'est-à-dire qu'elle porte une attention particulière à la complexité propre à chaque situation et n'hésite pas à entrer dans le détail des arrangements institutionnels bricolés par les communautés. L'élaboration théorique ne vient que dans un second temps, tentant de synthétiser la très grande quantité d'informations ainsi récoltées. Cette démarche est classique en science sociale, peut-être davantage en sociologie ou en anthropologie qu'en économie, mais l'importance de l'apport d'Ostrom se mesure à sa capacité à effectivement identifier les points clefs d'une gestion réussie des biens collectifs. La grille d'analyse qu'elle propose va en effet s'imposer et ne sera pas réellement remise en cause.

\subsection{Du terrain à la théorie: les conditions d'une gestion réussie des biens collectifs}

Son projet commence avec l'étude de la performance de groupes de policiers dans différents quartiers de la banlieue d'Indianapolis. Ensuite d'autres projets similaires vont suivre, dans les quartiers de Chicago et Saint-Louis, ce qui a ouvert la voie à une comparaison à grande échelle dans quatre vingt régions métropolitaines. La conclusion qui ressort de cette série d'études est que les services de police desservant les petits et moyens quartiers fonctionnent plus efficacement, et souvent à moindre coût, que les services de police desservant des quartiers de grandes tailles. "The presumption that economies of scale were prevalent was wrong; the 
presumption that you needed a single police department was wrong; and the presumption that individual departments wouldn't be smart enough to work out ways of coordinating is wrong" ${ }^{\prime \ddagger}$. Ostrom montre ainsi qu'en pratique l'idée, essentiellement théorique, selon laquelle il existerait des économies d'échelle est battue en brèche par l'analyse minutieuse des situations concrètes. Elle consacrera plus de quinze années à étudier les services de police mais n'hésitera pas, comme en témoigne la citation qui précède, à prendre clairement parti.

Par la suite, Ostrom réalisera d'autres études de cas qui lui permettront de conclure, dans son premier ouvrage "Gouverning the commons", que la gestion des ressources communes par les usagers est meilleure que prévue par la théorie standard. II s'agit notamment des études faites sur les forêts communales au Japon, en Suisse, sur les systèmes d'irrigation en Espagne, en Philippines ou au Sri-Lanka, sur la gestion des nappes phréatiques en Californie ou de la pêche en Turquie et en Ecosse. Au Sri-Lanka, l'organisation hiérarchique héritée du système traditionnel de politique locale, avait produit un système injuste et inefficace de distribution de l'eau. Ostrom montre comment le développement de nouvelles organisations collectives au niveau des agriculteurs a permis d'améliorer significativement la situation en modifiant les incitations et en fournissant un moyen de surveillance, améliorant aussi l'application des décisions collectives sur les détournements d'eau.

Ce qui frappe dans l'œuvre d'Ostrom c'est le nombre et la diversité des situations analysées. Pendant plusieurs décennies, l'atelier qu'elle anime se réunit une fois par semaine et passe en revue des exemples concrets de gestion des biens collectifs. II y a donc un aspect systématique dans son travail qui est rendu possible par l'implication autour de sa personne d'un véritable collectif de recherche.

\section{Principes fondamentaux de la gestion des biens collectifs}

"Governing the commons" constitue sans doute l'ouvrage le plus important d'Ostrom. Tout au long du texte, elle effectue un constant va et vient entre des descriptions minutieuses de cas concrets et des principes généraux. Elle identifie ainsi huit principes pour une gestion réussie de communs, qui fournissent les bases d'une théorie de la gestion des biens collectifs. Tout l'art d'Ostrom réside dans sa capacité

\footnotetext{
${ }^{\ddagger}$ Ostrom and Whitaker (1997), pp: 48-76
} 
à fonder chacune de ses affirmations sur différents exemples montrant comment l'adhésion, ou non, à l'un des principes, suffit à changer le cours des choses. Rappelons brièvement ces principes.

1. les droits d'accès doivent être clairement définis

2. les avantages doivent être proportionnels aux coûts assumés

3. des procédures doivent être mise en place pour faire des choix collectifs

4. des règles de supervision et surveillance doivent exister ;

5. des sanctions graduelles et différenciées doivent être appliquées

6. des mécanismes de résolution des conflits doivent être institués

7. l'Etat doit reconnaître l'organisation en place ;

8. l'ensemble du système est organisé à plusieurs niveaux.

Ces principes débordent du cadre habituel de l'économie pour inclure des aspects juridiques, sociaux et politiques. En effet, si les deux premiers principes paraissent familiers aux économistes, l'application de règles de supervision et de surveillance ressort plutôt du cadre socio-juridique, de même que la mise en place de mécanismes de résolution des conflits.

Ces principes révèlent que les structures institutionnelles sont gérées dans un système polycentrique (nested institutions). En effet, les principes 1 et 2 régissent les règles d'usages individuels alors que les principes 3,4 et 5 sont plutôt d'ordre collectif. Le septième principe montre que l'Etat influe sur l'action collective, sans pour autant être directement acteur de la gestion.

Au regard des théories économiques, le point peut-être le plus important concerne l'importance de laisser les acteurs discuter entre eux pour élaborer en commun leurs propres règles de fonctionnement. Comme le remarque justement Ostrom, la théorie économique est muette sur le type de règle qui peut émerger d'une discussion entre individus appartenant à une même collectivité. De plus, la communication entre individus est un facteur essentiel dans le développement de relations de confiance entre individus. Là encore, la théorie économique actuelle ne fournit guère d'éléments permettant de décrire les formes institutionnelles favorisant l'émergence de la confiance. 


\subsection{De la théorie à l'action publique}

Ostrom sera l'une des premières à faire usage de l'économie expérimentale pour simuler et évaluer l'impact d'une modification des règles régissant la gestion des biens collectifs En ce sens, elle note que "the use of economic experiments has enhanced the already diverse knowledge from theoretical and field sources of when and how groups can solve the problem through selfgoverning echanisms" (Cardenas and Ostrom (2001)). Ainsi, dans l'une de ses expériences typiques, un certain nombre de sujets interagissent pendant plusieurs périodes sans savoir exactement laquelle sera la dernière. A chaque période, chaque sujet peut contribuer à un bien public. Le coût marginal de la contribution de chaque individu est supérieur à son bénéfice marginal mais plus petit que le bénéfice total. Dans ce contexte, un individu égoïste et rationnel refusera de contribuer au bien collectif. L'une des principales caractéristiques de ces expériences est l'introduction de possibilités de sanction. Dans un traitement, chaque sujet est informé de l'action des autres sujets lors du tour précédent et peut punir sélectivement chacun des adversaires. Cependant, punir est coûteux. Ostrom et ses collègues constatent pourtant que beaucoup de sujets utilisent la possibilité, coûteuse pour eux, de sanctionner les comportements déviants. De plus ces punitions sont beaucoup plus efficaces si les sujets sont autorisés à communiquer que s'ils ne le sont pas, ce qui conforte ses résultats obtenus sur le terrain. Au final, des dispositifs institutionnels simples suffisent en pratique à obtenir une bonne gestion des biens publics, y compris dans des situations où les théories fondées sur l'hypothèse d'individus égoïstes prévoit une destruction totale du bien collectif. Les résultats tirés de ses expériences sont publiés dans son ouvrage coécrit avec Gardner et Walker, "Rules, Game and Common-Pool Ressources"(1994). Ces expériences ont été suivi par d'autres notamment celles de Masclet et al. (2003), Isaac and Walker (1988), Schlager (1994), Kosfeld and Ried (2009) ou celles Fehr and Gächter (2000) sur des possibilités de sanctions à des

jeux non répétés. Ces travaux ont confirmé les conclusions d'Ostrom : un grand nombre d'individus est prêt à subir une perte financière pour punir un comportement déviant. 
Au plan méthodologique, le recours à l'économie expérimentale permet d'étudier la réaction des communautés à des modifications de leur environnement institutionnel. En cela, l'économie expérimentale permet de passer d'une description des situations de coopération - via des études de cas - à l'étude des mesures à mettre en oeuvre pour améliorer les pratiques en cours.

\section{Un modèle d'analyse: I'Institutional Analysis and Development (IAD)}

Le modèle IAD est utilisé depuis plus de trois décennies comme grille d'analyse des situations de gestion commune. Apparu pour la première fois en 1982 dans Kiser and Ostrom (1982), la description la plus explicite du modèle IAD a été faite en 1994 par Ostrom dans son ouvrage "Rules, Game and Common-Pool Ressources" (Ostrom et al. (1994)).

L'analyse commence sur une unité conceptuelle appelée scène d'action (Action Arena) regroupant des acteurs qui interagissent dans un espace social appelé situation d'action (action situation) (figure 1). Une situation d'action renvoie à l'espace social où les participants dotés de diverses préférences interagissent, échangent des biens et services, résolvent des problèmes, se dominent, ou se battent (Ostrom (2005)). Après la délimitation de la structure initiale, Ostrom distingue les facteurs externes qui affectent la scène d'action. "The IAD framework is thus a general language about how rules, physical and material conditions, and attributes of community affect the structure of action arenas, the incentives that individuals face, and the resulting outcomes" (Ostrom (1999)). Ainsi, les facteurs affectant la structure d'une scène d'action comprennent trois ensembles de variables qui sont les caractéristiques du monde physique, les caractéristiques de la communauté et les règles en vigueur. 


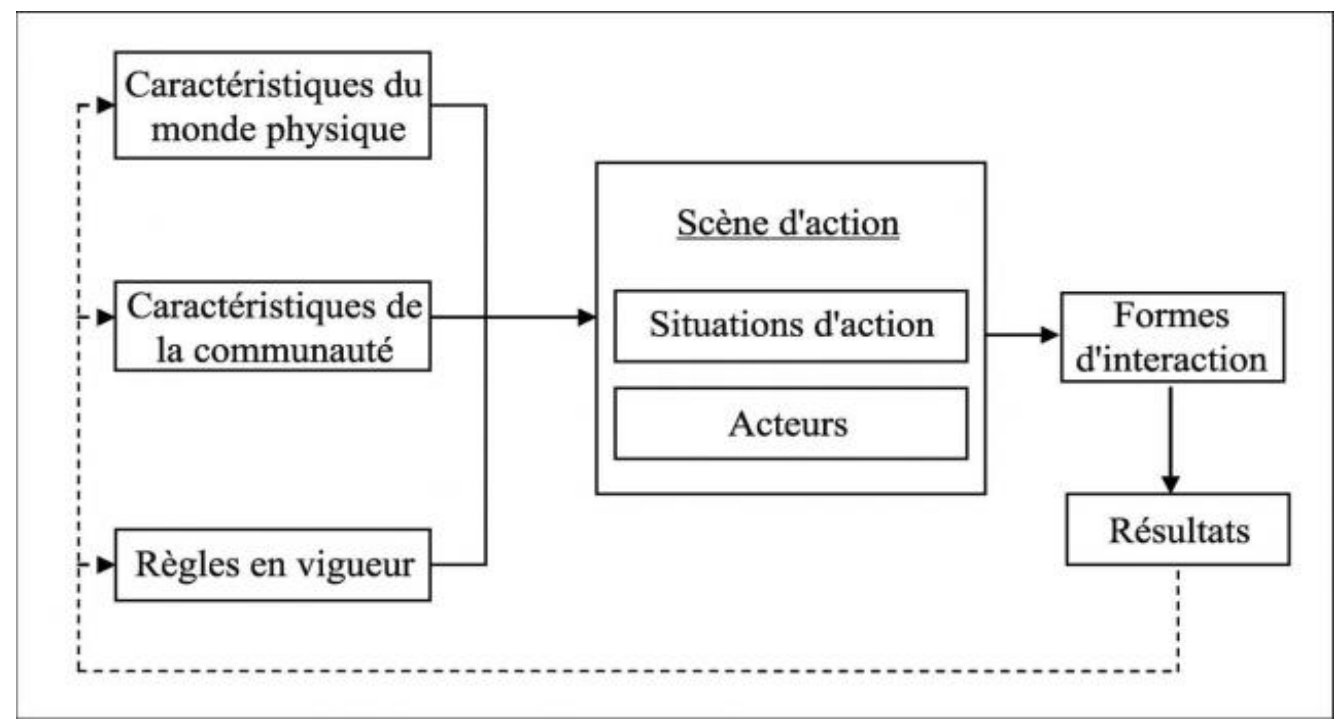

Figure 1. Modèle IAD (Ostrom et al.,1994)

- Le monde biophysique à laquelle l'action est liée (par exemple une pêcherie ou une forêt) est l'une des variables critiques qui affectent la structure de la situation d'action. A l'évidence la problématique du réchauffement climatique n'est pas la même que celle de la gestion d'un étang.

- Les principaux attributs d'une communauté qui affectent l'arène d'action sont: les normes de comportement généralement accepté dans la communauté, le niveau de compréhension commune, le niveau d'homogénéité dans les préférences de ceux qui vivent dans une communauté, la taille et la composition de la communauté concernée, etc.

- Les règles constituent le principal levier d'action puisqu'elles sont susceptibles d'être modifiées à court terme. Mais surtout, les règles sont souvent identifiées par les acteurs eux-mêmes comme le principal vecteur du changement. Résoudre une situation conflictuelle passe prioritairement, aux yeux des acteurs, par l'établissement de nouvelles règles.

Le modèle IAD doit sans doute être compris comme un cadre opérationnel, une grille de lecture, servant de base à une évaluation des problèmes de gestion commune. En d'autres termes, il ne s'agit pas de proposer un modèle théorique, ni une description simplifiée de ce qui se passe en pratique sur le terrain. L'apport du modèle IAD est de fixer un langage et de pointer en direction des problèmes attendus. L'évaluation d'un schéma aussi simple que celui présenté ci-dessus doit 
donc se faire au regard de sa capacité pratique à guider les personnes en charge d'une évaluation. En ce sens, le modèle IAD est un succès puisqu'il constitue aujourd'hui la matrice de base des études portant sur les biens collectifs. Son usage est fortement recommandé par nombre d'organismes internationaux. (Pour des exemples d'utilisation du modèle IAD voir: Tang (1992); Lam (1998); Andersson and Ostrom (2008); Yandle (2001); Ostrom (1992); Oakerson (1992); Polski and Ostrom (1998))

\section{Conclusion: vers une vision renouvelée de la rationalité ?}

Pour Ostrom, l'objet de l'action collective est la résolution de dilemmes sociaux résultant de la gestion de biens collectifs. Ostrom reconnaît que la tentation de resquiller est effectivement constatée dans la pratique mais elle souligne que ces tendances néfastes ne suffisent pas pour annihiler toute possibilité de parvenir à une gestion efficace. Ostrom en tire une critique, frontale et assumée, des théories de la rationalité souvent utilisées en économie pour décrire les comportements individuels. Pour elle, "l'hypothèse de rationalité des théories de la " première génération " a été rejetée à plusieurs reprises par les recherches empiriques menées sur le terrain et au laboratoire " (Ostrom (1998)). Les individus ne calculent pas un ensemble complet de stratégies pour toutes les situations qu'ils rencontrent et d'ailleurs, peu de situations dans la vie génèrent l'information sur toutes les actions possibles que l'on peut prendre, sur tous les résultats qui peuvent être obtenus ou sur toutes les stratégies que les gens peuvent prendre. Pour Ostrom, "la seule hypothèse raisonnable que l'on peut formuler sur les processus de calcul et de découverte est que les acteurs sont engagés dans un apprentissage fondé sur un grand nombre d'essais et d'erreurs"(Ostrom (1990)). On peut voir les travaux d'Ostrom comme un défi pour les théories de la rationalité. II ne s'agit pas de rejeter en bloc toute tentative de formalisation du comportement humain mais plutôt de pointer les insuffisances des modèles actuels. Deux exemples sont emblématiques cet égard.

- Le rôle de la communication: Pour Ostrom, la communication est fondamentale dans l'action collective. Une communication directe est le fondement même d'une gestion collective réussie. Elle favorise la confiance et permet d'élaborer des règles en commun. Ce qui se joue véritablement dans ces échanges verbaux échappe 
encore largement aux théories contemporaines mais trouve un large écho auprès des praticiens (pour une discussion plus détaillée de ce point voir Hollard (2004)).

-L'importance de l'innovation institutionnelle, des règles et sanctions: D'après Ostrom, "dans tous les régimes d'auto-gouvernance des ressources communes connus qui ont survécu pendant plusieurs générations, les participants ont investi des ressources dans la surveillance et la sanction des actions des uns et des autres de façon à réduire la probabilité du free riding" (Ostrom, 1990). Qu'il faille instaurer des sanctions et une surveillance pour éviter une utilisation abusive de la ressource n'est pas en soi un résultat nouveau ou surprenant. Ce qu'il l'est plus c'est que la charge de la surveillance est assumée par les acteurs eux-mêmes. Les activités de surveillance deviennent ainsi elles-mêmes un bien collectif. Le fait que les acteurs eux-mêmes assurent la surveillance, et non pas l'Etat pas exemple, est fondamental. Ostrom n'hésite pas à affirmer, par exemple lors de la leçon donnée lors de la réception du Nobel, que cette implication des acteurs dans les activités de surveillance est plus importante que la définition des droits de propriétés sur la ressource.

Les grandes théories de l'organisation sociale, mettant par exemple l'Etat ou le marché au centre de la scène, reposent souvent sur un schéma où chaque acteur est confronté à des dispositifs institutionnels exogènes. Les acteurs font face à des lois ou des prix qu'ils n'ont pas contribués à façonner. Les groupes de petite taille peuvent cependant agir collectivement de manière à générer eux-mêmes des dispositifs institutionnels qui leur soient propres. Au fond, ce qui importe pour Ostrom n'est pas tellement la nature des règles qui seront mises en œuvre mais plutôt l'implication des acteurs dans l'élaboration et la surveillance du bon respect des règles. Le fait qu'il n'existe pas de théorie complète de ces processus d'autogestion, ne doit pas faire oublier qu'il existe des contextes dans lesquels ils se révèlent très supérieurs aux formes d'organisations les mieux conceptualisées. 


\section{Références}

- Andersson, K. and E. Ostrom (2008). Analyzing decentralized resource regimes from a polycentric perspective. Policy Sciences (41), 71-93.

- Cardenas, J. and E. Ostrom (2001). What do people bring into the game? how normes help overcome the tragedy of the commons. 4eme Journées d'Economie de l'Environnement de Toulouse.

- Coase, R. (1960). The problem of social cost. Journal of Law and Economics 3,1-44.

- Fehr, E. and S. Gächter (2000). Cooperation and punishment in public goods experiments. American Economic Review (90), 980-994.

- Gordon, H. (1957). The economic theory of a common property resource: The fishery. Journal of Political Economic 62, 124-142

- Hardin, G. (1968). The Tragedy of the Commons. Science.

- Hollard, G. (2004). La délibération dans la théorie économique. Cahiers d'économie politique "Les économistes et la démocratie ; Qu'a-t-on appris depuis Schumpeter? " 47, 173-190.

- Isaac, R. and J. Walker (1988). Communication and free-riding behavior: The voluntary contribution mechanism. Economic Inquiry (26).

- Kiser, L. et E. Ostrom (1982). Strategies of Political Inquiry (Ostrom, Elinor ed.), Chapter: The Three Worlds of Action, A Metatheoretical Synthesis of Institutional Approaches. Beverly Hills: Sage.

- Kosfeld, M. Okada, A. and A. Ried (2009). Institution formation in public goods games. American Economic Review, (99), 1335-1355.

- Lam, W. (1998). Governing Irrigation Systems in Nepal: Institutions, Infrastructure, and Collective Action. ICS Press, Oakland.

- Masclet, D., Noussair, S. Tucker, and M. Villeval (2003). Monetary and nonmonetary punishment. American Economic Review (93,), 366-380.

- Oakerson, R. (1992). Making the commons work: theory practice and policy (Bromley D.W. ed.), Chapter Analysing the Commons: A Framework, pp. 41-59. San Francisco, California, Institute for Contemporary Studies.

- Ostrom, E. (1965). Public Entrepreneurship: A Case Study in Ground-Water Basin Management. Ph. D. thesis, University California Los Angeles. 
- Ostrom, E. (1990). Governing the Commons: The Evolution of Institutions for Collective Actions. Cambridge: Cambridge University Press.

- Ostrom, E. (1992). Crafting Institutions for Self-Governing Irrigation Systems. San Francisco.

- Ostrom, E. (1998). A behavioral approach to the rational choice theory of collective action: Presidential address, american political science association, 1997. American Political Science Review, 1-22.

- Ostrom, E. (1999). In Theories of the Policy Process (Paul A. Sabatier ed.), Volume 2, Chapter Institutional Rational Choice: An Assessment of the Institutional Analysis and Development Framework, pp. 493-535. Boulder, CO: Westview Press.

- Ostrom, E. (2005). Understanding Institutional Diversity. Princeton University Press.

- Ostrom, E., R. Gardner, and J. Walker (1994). Rules, Games, and CommonPool Resources. Ann Arbor: University of Michigan Press..

- Ostrom, E. and G. Whitaker (1997). Does local community control of police make a difference? Some preliminary findings. Journal of Political Science 17, 48-76.

- Pigou, A. C. (1920). The Economics of Welfare. New York: Macmillan.

- Polski, M. and E. Ostrom (1998). An Institutional Framework for Policy Analysis and Design. Bloomington.

- Schlager, E. (1994). Rules, Games, and Common-pool Resources (E. Ostrom, R. Gardner and J.Walker ed.)., Chapter Fishers' Institutional Responses to Common-pool Resource Dilemmas, pp. 247-66. University of Michigan Press.

- Tang, S. (1992). Institutions and Collective Action: Self-Governance in Irrigations. ICS Press San Francisco.

- Warming, J. (1911). On the rent of fishing grounds. History of Political Economy 15, 391-396.

- Yandle, B. (2001). Companion to Public Choice; (Shughart, William f. And Razzolini, Laura ed.), Chapter The Elgar Public Choice and the environment. Cheltenham: Edward Elgar. 